\title{
Plantio de árvores de crescimento rápido para recuperação de áreas agrícolas na Amazônia Oriental brasileira: estudo de caso com produção de milho e mandioca
}

\author{
Silvio Brienza Júnior ${ }^{1}$, Raimundo Parente de Oliveira ${ }^{2}$, Manfred Denich ${ }^{3}$, Paul L. G. Vlek \\ 1Embrapa Amazônia Oriental, CP 48, CEP 66095-100, Belém, PA, Brasil \\ ${ }^{2}$ Cooperativa de Economia e Crédito Mútuo do Pessoal do Ministério da Agricultura, Pecuária e Abastecimento no Estado do Pará. Trav. Pirajá, 1966, \\ CEP 66095-100, Belém, PA, Brasil \\ ${ }^{3}$ Centro para o Desenvolvimento de Pesquisa, Universidade de Bonn. Walter- Flex-Str. 3, D-53113, Bonn, Alemanha
}

"Autor correspondente:

brienza@cpatu.embrapa.br

Termos para indexação:

Enriquecimento de capoeira

Espaçamento de plantio

Área degradada

Árvores leguminosas

Index terms:

Fallow enrichment

Density of planting

Degraded land

Leguminous tree

Histórico do artigo:

Recebido em 05 set 2011

Aprovado em 19 dez 2011

Publicado em 28 dez 2011

doi: 10.4336/2011.pfb.31.68.347
Resumo - O objetivo do presente trabalho foi avaliar a sobrevivência de árvores leguminosas plantadas para recuperar áreas degradadas e as produtividades de milho e mandioca num sistema tradicional de agricultura de derruba-e-queima. Foram utilizados os espaçamentos $1 \mathrm{~m} \times 1 \mathrm{~m}, 2 \mathrm{~m} \times 1 \mathrm{~m} \mathrm{e} 2 \mathrm{~m} \times 2 \mathrm{~m}$ para plantio de Acacia angustissima, Clitoria racemosa, Inga edulis e Acacia mangium. A espécie Sclerolobium paniculatum foi plantada somente no espaçamento $2 \mathrm{~m}$ x $1 \mathrm{~m}$. Na condução do experimento, o milho permaneceu no experimento nos primeiros quatro meses e considerado como monocultura, produziu $1.890 \pm 32 \mathrm{~kg} \mathrm{ha}^{-1}$. A sobrevivência das árvores, plantadas junto com milho e na mesma época da mandioca, não sofreu interferência negativa das culturas agrícolas. Independente do espaçamento, as árvores não causaram impacto negativo no peso seco das raízes de mandioca. Quanto a densidade de árvores observouse tendência de aumento do peso seco das raízes de mandioca com a diminuição do número de árvores plantadas por hectare. Os resultados evidenciam a possibilidade de se manter a produtividade agrícola associada ao plantio de árvores. Entretanto, a opção pelo espaçamento $1 \mathrm{~m}$ x $1 \mathrm{~m}$ deve ser cuidadosamente analisada quando o objetivo principal for a produção de alimentos.

\section{Planting of fast-growing trees for recovery of agricultural areas in the Eastern of Brazilian Amazon: studying case of maize and cassava prodution}

\begin{abstract}
The objective of the present paper was to evaluate the survival of planted leguminous trees to restore degraded areas and yields of maize and cassava in traditional farming system. The trees species Acacia angustissima, Clitoria racemosa, Inga edulis, and Acacia mangium were planted at spacing of $1 \mathrm{~m} \times 1 \mathrm{~m}, 2 \mathrm{~m} \times 1 \mathrm{~m}$ and $2 \mathrm{~m} \times 2$ $\mathrm{m}$. Sclerolobium paniculatum was planted only at spacing of $2 \mathrm{~m} \times 1 \mathrm{~m}$. The maize, remained in the cropping system during the first four months, and it was considered as monocropping phase and, yielded $1,890 \pm 32 \mathrm{~kg} \mathrm{ha}^{-1}$. The survival of planted trees was not negatively impacted by the presence of agricultural crops. Regardless of the spacing, the planted trees did not cause a negative impact on dry weight of cassava root. There was a tendency to increase dry weight of root of cassava as the number of planted trees per hectare decreased. These results show the possibility to maintain crop productivity in enriched fallow vegetation systems. However, tree planting at the spacing of $1 \mathrm{~m} \mathrm{x}$ $1 \mathrm{~m}$ should be carefully considered when food is required.
\end{abstract}




\section{Introdução}

As iniciativas de desenvolvimento praticadas na Amazônia brasileira têm subestimado a complexidade biológica do ambiente amazônico e a real potencialidade de seus recursos naturais. Na prática, observa-se uma ocupação desordenada da terra, resultando quase sempre no desenvolvimento de atividade ecológica e/ ou sócioeconomicamente não sustentável. A agricultura baseada na prática de derruba-e-queima tem sido considerada um dos agentes causadores de degradação ambiental na Amazônia brasileira devido a sua forma itinerante. A maior crítica dessa prática deve-se ao fato de áreas de florestas serem derrubadas para o cultivo agrícola visando à subsistência do agricultor. Essa forma de agricultura ainda utiliza tecnologia rudimentar e oferece pouca oportunidade para o agricultor acumular capital e melhorar seu padrão de vida. A vegetação denominada de pousio (capoeira) que surge após os cultivos agrícolas é importante para o acúmulo de biomassa vegetal e restabelecimento do ciclo biogeoquímico de nutrientes. Fatores como o crescimento populacional e a divisão do lote familiar com consequente intensificação do seu uso (Silva et al., 1998) contribuem para o encurtamento do tempo de pousio. E, como resultado disso, são constatadas perdas de nutrientes devido ao tempo insuficiente para os acúmulos de biomassa e nutrientes pela capoeira (Hölscher, 1995). Esse quadro provoca instabilidade no uso da terra e contribui para o crescimento de áreas abandonadas, podendo levar a agricultura familiar ao colapso. Por exemplo, cerca de $20 \%$ das áreas desmatadas na Amazônia encontram-se abandonadas ou subutilizadas ou muitas vezes em estado de degradação (Almeida et al., 2010). A busca de sistemas produtivos mais adequados às condições edafoclimáticas da Amazônia deve contemplar práticas sustentáveis de conservação ambiental, dos pontos de vista agronômico, ambiental, social e econômico, além da necessidade de garantir sua replicação no tempo. Algumas tecnologias agrícolas como sistema bragantino (Galvão et al., 2008), preparo de área sem queima (Kato et al., 2007) e manejo do solo com leguminosas (Lopes \& Galeão, 2006) já foram desenvolvidas no sentido de melhorar a produtividade da agricultura familiar. $\mathrm{O}$ enriquecimento de capoeira a partir do plantio de árvores leguminosas de crescimento rápido, é outra alternativa tecnológica para promover acúmulos de biomassa e nutrientes (Brienza Júnior, 1999). Esse processo, além de possibilitar manter um curto período de pousio, pode produzir uma vegetação secundária semelhante a encontrada em capoeiras velhas (Brienza Júnior, 1999). O objetivo do presente trabalho foi avaliar a sobrevivência de cinco espécies arbóreas e as produções de milho e mandioca num sistema de produção agrícola onde árvores foram plantadas para o enriquecimento da capoeira durante a fase de cultivo agrícola.

\section{Material e métodos}

$\mathrm{O}$ experimento foi implantado em uma propriedade de agricultor familiar, localizada na região Bragantina, no Município de Igarapé-açu, PA (01 $07^{\circ} 33^{\prime}$ ' Sul e $\left.47^{\circ} 37^{\prime} 27^{\prime \prime} \mathrm{W}\right)$, baseado no sistema de produção tradicional de milho-mandioca. A vegetação de pousio da área experimental apresentava de quatro a cinco anos de idade. Os detalhes de solo, clima e paisagem são encontrados em Denich (1989), Watrin (1994), Vieira (1996) e Brienza Júnior (1999). O preparo da área foi tradicional, envolvendo derruba-e-queima da vegetação de pousio. Foram utilizadas 68 parcelas experimentais de $10 \mathrm{~m}$ x $8 \mathrm{~m}$. O plantio de milho (janeiro de 1995), variedade BR 106, obedeceu ao espaçamento de 1,0 $\mathrm{m} \times 0,5 \mathrm{~m}$. Por ocasião do plantio foram aplicados os equivalentes a $200 \mathrm{~kg} \mathrm{ha}^{-1}$ do adubo NPK na formulação 10-28-20 e 35 dias depois $70 \mathrm{~kg} \mathrm{ha}^{-1}$ de uréia e $30 \mathrm{~kg}$ ha $^{-1}$ de $\mathrm{KCl}$ (Souza et al., 1999). A colheita do milho ocorreu com 120 dias e após foram obtidos os pesos secos de grãos, de sabugo, de palha e total de espigas. A mandioca, variedade "olho verde", foi plantada sem adubação (fevereiro de 1995) no espaçamento de $1 \mathrm{~m} \mathrm{x}$ $1 \mathrm{~m}$, e sua colheita ocorreu aos 12 meses de idade, com posterior obtenção dos pesos de matéria seca de raiz e produção de raiz fresca. As espécies arbóreas Acacia angustissima Kuntze (ligeirinha), Clitoria racemosa $\mathrm{G}$. Don (palheteira), Inga edulis Mart. (ingá), Sclerolobium paniculatum Vogel (taxi-branco) e Acacia mangium Willd. (acácia) foram plantadas, após a colheita do milho em junho de 1995, nos espaçamentos $1 \mathrm{~m} \mathrm{x} 1 \mathrm{~m}$ (10 mil árvores ha $\left.{ }^{-1}\right), 2 \mathrm{~m}$ x $1 \mathrm{~m}$ (5 mil árvores ha $\left.{ }^{-1}\right)$ e 2 $\mathrm{m}$ x $2 \mathrm{~m}$ (2.500 árvores ha-1 ${ }^{-1}$. A espécie taxi-branco foi plantada apenas no espaçamento $2 \mathrm{~m}$ x $1 \mathrm{~m}$. No sistema adotado, árvores e mandioca cresceram juntos durante oito meses até a colheita das raízes de mandioca. Os valores de sobrevivência das espécies arbóreas referemse a 12 meses de idade. $\mathrm{O}$ experimento obedeceu ao delineamento estatístico de blocos ao acaso com quatro repetições. Os dados das variáveis estudadas 
foram submetidos a análise de variância (ANOVA). Para obtenção da distribuição normal os valores de porcentagem de sobrevivência foram transformados para Arc Sen e posterior análise. A avaliação dos tratamentos foi feita usando ANOVA, considerando o modelo fatorial completo (espécies arbóreas, espaçamento de plantio e interações). Posteriormente, o tratamento controle (sem plantio de árvores) foi comparado com as espécies arbóreas, independente da densidade de plantio e vice-versa. O teste LSD $(p<0,05)$ foi utilizado para comparação de médias em casos apropriados.

\section{Resultados e discussão}

\section{Sobrevivência das árvores plantadas}

A porcentagem de sobrevivência de uma espécie reflete a sua capacidade de adaptação às condições ambientais onde é plantada. A ANOVA realizada a partir dos valores médios de sobrevivência, aos 12 meses de idade, considerando o modelo fatorial completo (bloco, árvores leguminosas e densidade de plantio) e sem a espécie taxi-branco (não incluída devido ao seu plantio somente no espaçamento $2 \mathrm{~m} \times 1 \mathrm{~m}$ ), mostrou diferença significativa apenas para espécies leguminosas $\left(\mathrm{F}_{145,70}=10.8182 ; \mathrm{p}<0,000\right)$ (Tabela 1). A. mangium apresentou a menor taxa de sobrevivência $(91,6 \%)$ quando comparada com as demais espécies estudadas (Tabela 1). A sobrevivência das árvores plantadas para enriquecimento não apresentou dependência do espaçamento de plantio $\left(\mathrm{F}_{0,64}=0.0480 ; \mathrm{p}<0,953\right)$, mesmo quando um maior número de árvores por hectare ( espaçamento $1 \mathrm{mx} 1 \mathrm{~m}$ ) foi considerado (Tabela 2).

Tabela 1. Taxa média de sobrevivência (\%) aos 12 meses de idade, para as leguminosas arbóreas $A$. angustissima, $C$. racemosa, I. edulis e $A$. mangium plantadas para enriquecer a vegetação de pousio, independentemente do espaçamento de plantio $1 \mathrm{~m} \times 1 \mathrm{~m}, 2 \mathrm{~m} \times 1 \mathrm{~m}$ e $2 \mathrm{~m} \times 2 \mathrm{~m}$.

\begin{tabular}{lcc}
\hline \multicolumn{1}{c}{ Espécies florestais } & $\mathbf{n}$ & Sobrevivência (\%) \\
\hline C. racemosa & 12 & $99,2 \mathrm{a}$ \\
A. angustissima & 12 & $96,9 \mathrm{a}$ \\
I. edulis & 12 & $97,1 \mathrm{a}$ \\
A. mangium & 12 & $91,6 \mathrm{~b}$ \\
\hline
\end{tabular}

Dados seguidos da mesma letra não diferem estatisticamente; Teste LSD $(\mathrm{p}<0,05)$.
Tabela 2. Médias de sobrevivência (\%), aos 12 meses de idade, obtidas para os espaçamentos $1 \mathrm{~m}$ x $1 \mathrm{~m}, 2 \mathrm{~m}$ x $1 \mathrm{~m} \mathrm{e}$ $2 \mathrm{~m} \times 2 \mathrm{~m}$, independentemente das árvores leguminosas $A$. angustissima, C. racemosa, I. edulis e A. mangium plantadas para enriquecer a vegetação de pousio.

\begin{tabular}{ccc}
\hline Espaçamento plantio & $\mathbf{n}$ & Sobrevivência (\%) \\
\hline $1 \mathrm{~m} \times 1 \mathrm{~m}$ & 16 & $95,5 \mathrm{a}$ \\
$2 \mathrm{~m} \times 1 \mathrm{~m}$ & 16 & $96,3 \mathrm{a}$ \\
$2 \mathrm{~m} \times 2 \mathrm{~m}$ & 16 & $96,9 \mathrm{a}$ \\
\hline
\end{tabular}

Dados seguidos da mesma letra não diferem estatisticamente; Teste LSD $(\mathrm{p}<0,05)$.

Todas as espécies arbóreas estudadas apresentaram baixos percentuais de mortalidade, mostrando que é possível associar seus plantios à fase agrícola. A taxa de sobrevivência observada acima de $90 \%$ é compatível com a recomendação da FAO (1981) para plantios comerciais de Eucalyptus sp. A taxa de sobrevivência observada para $S$. paniculatum foi de $83,8 \%(\mathrm{n}=4)$. Esse valor é superior aos 77,8\% observados por Castro et al. (1998) para o taxi-branco plantado em espaçamentos variando entre $2,0 \mathrm{~m}^{2}$ e 7,5 $\mathrm{m}^{2}$ por planta, em Macapá, AP.

Durante a fase de estabelecimento do experimento foram identificadas duas fases importantes em relação à taxa de sobrevivência das espécies plantadas. A primeira fase refere-se à produção de mudas. Mudas de boa qualidade e rustificadas apresentam maiores chances de adaptação às condições de campo, no momento do plantio. A segunda fase diz respeito ao período em que as árvores se desenvolvem em conjunto com a cultura da mandioca. Nessa fase, as atividades de capina devem ser realizadas cuidadosamente para não causar danos mecânicos às árvores plantadas. Esse fato é, particularmente, um problema quando as espécies utilizadas são desconhecidas para o agricultor.

Trabalhos de enriquecimento da vegetação de pousio, realizados na Amazônia brasileira, apresentaram taxas de sobrevivência comparáveis às observadas no presente trabalho. No método recrû (Catinot, 1965) utilizado para o enriquecimento da vegetação de pousio, o objetivo principal é a produção de madeira em rotações de 25-30 anos. As árvores plantadas para enriquecer a vegetação de pousio, de acordo com este método, apresentaram taxas de sobrevivência que variaram de $87 \%$ a $100 \%$ (Yared \& Carpanezzi, 1981). Na região do Tapajós, PA, o enriquecimento da vegetação de pousio também foi testado com base no método Taungya (King, 1968), em que as árvores foram plantadas durante o período 
de cultivo e no final do período agrícola. Nesse método as árvores crescem até a colheita (entre 25-30 anos, dependendo da espécie). As taxas de sobrevivência das espécies florestais estudadas nessas condições variaram de $70 \%$ a $100 \%$ (Brienza Júnior, 1982; Brienza Júnior et al., 1983). Em outro estudo na região do Tapajós, envolvendo o consórcio de espécies madeireiras e diferentes culturas agrícolas (anuais e perenes), as taxas de sobrevivência das árvores variaram de $20 \%$ a $100 \%$ (Marques et al., 1993).

Yared et al. (1988) observaram taxas de sobrevivência de $97 \%, 95 \%$ e $95 \%$, respectivamente, para as espécies nativas Laetia procera (66 meses de idade), S. paniculatum (66 meses de idade ) e Jacaranda copaia (78 meses de idade) plantadas em áreas abertas na Amazônia brasileira. Gonzalez \& Fisher (1994), estudando o crescimento de várias árvores plantadas em pastagens abandonadas na Costa Rica, observaram valores de sobrevivência de $86 \%$ e $92 \%$, respectivamente para A. mangium e I. edulis.

\section{Produção de milho}

AANOVA usando o modelo fatorial completo (bloco, árvores leguminosas e densidade de árvores plantadas) não mostrou diferenças significativas para as produções de grão $\left(\mathrm{F}_{106293,6}=3123,5 ; \mathrm{p}<0,9272\right)$, de sabugo $\left(\mathrm{F}_{3123,5000}=0,2004 ; \mathrm{p}<0,9375\right)$, de palha $\left(\mathrm{F}_{6643,1000}=0,2004\right.$; $\mathrm{p}<0,8821)$ e total de espigas de milho $\left(\mathrm{F}_{191195,0}=0,2106\right.$; $\mathrm{p}<0,9319$ ). Na execução do processo de plantar espécies florestais durante a fase agrícola, o milho permaneceu junto com as árvores durante os primeiros quatro meses do sistema. Ou seja, as árvores estavam no estágio de muda quando o milho foi colhido. Por isso não se observou influência da presença das árvores sobre a cultura do milho. Os resultados referentes às varáveis analisadas para o milho são apresentados na Tabela 3 .

Tabela 3. Médias ( \pm erro padrão) e coeficientes de variação (CV) para os pesos secos de milho referentes a grão, sabugo, palha e total.

\begin{tabular}{ccccc}
\hline & \multicolumn{4}{c}{ Produção $\left(\mathbf{k g ~ h a}^{-1}\right)$} \\
\cline { 2 - 5 } Média & Grão & SABUGo & PALHA & Total \\
\cline { 2 - 5 } CV (\%) & $1.890 \pm 32$ & $360 \pm 5$ & $440 \pm 8$ & $2.690 \pm 43$ \\
\hline
\end{tabular}

A produtividade de milho encontrada foi comparável à obtida em estudo similar $\left(1.700 \mathrm{~kg} \mathrm{ha}^{-1}\right)$ realizado com Acacia auriculiformis (Silva Júnior et al., 1998). E, por outro lado, em experimento tradicional de milho no sudeste do Estado do Pará, usando-se adubação de 150 $\mathrm{kg} \mathrm{ha}^{-1}$ de NPK 10-28-20, a mesma cultivar do presente trabalho produziu $1.550 \mathrm{~kg} \mathrm{ha}^{-1}$ de grãos (Alves et al., 2001).

\section{Produção de mandioca}

Durante o desenvolvimento do sistema de enriquecer a capoeira com o plantio de espécies arbóreas a partir da fase agrícola, as árvores plantadas e a mandioca permaneceram juntas por oito meses, até a colheita da cultura agrícola. Os parâmetros avaliados para a mandioca foram produção de matéria seca de raiz e produção de raiz fresca. A análise de variância usando o modelo fatorial completo (bloco, árvores leguminosas e densidade de árvores plantadas) não mostrou diferenças significativas para os fatores espécie arbórea e interação espécie arbórea $\mathrm{x}$ densidade de plantio. Somente o fator densidade de plantio de árvores para enriquecimento $\left(\mathrm{F}_{1303097,80}=9,13\right.$; $\mathrm{p}<0,0001$ para peso seco e $\mathrm{F}_{9912668,35}=9,13 ; \mathrm{p}<0,01$ para peso fresco) foi significativo.

O enriquecimento da capoeira com diferentes espécies de árvores, independente do espaçamento de plantio, não causou impacto negativo na produção de peso seco de raiz de mandioca. $\mathrm{O}$ maior valor de peso seco de raiz de mandioca $\left(7.120 \pm 350 \mathrm{~kg} \mathrm{ha}^{-1}\right)$ foi observado na presença de $S$. paniculatum, enquanto que o menor $(6.100 \pm$ $290 \mathrm{~kg} \mathrm{ha}^{-1}$ ) com C. racemosa (Tabela 4). Embora não tenham sido observadas diferenças estatísticas entre as espécies, e entre as espécies e o controle $\left(6.060 \pm 780 \mathrm{~kg} \mathrm{ha}^{-1}\right)$, todos os valores de pesos secos obtidos na presença das árvores foram maiores que o controle. Assim, pode-se comentar que o uso de leguminosas para enriquecer a vegetação de pousio não interferiu negativamente na produção de peso seco de mandioca e, consequentemente, na produção de farinha de mandioca que o agricultor pode obter. 
Tabela 4. Médias ( \pm erro padrão) e coeficientes de variação (CV) dos pesos secos de raízes de mandioca obtidos em consórcio com diferentes leguminosas arbóreas plantadas para enriquecimento da vegetação de pousio nos espaçamentos $1 \mathrm{~m} \times 1 \mathrm{~m}, 2 \mathrm{~m} \times 1 \mathrm{~m}$ e $2 \mathrm{~m} \times 2 \mathrm{~m}$.

\begin{tabular}{lrcc}
\hline \multicolumn{1}{c}{$\begin{array}{c}\text { Sistema de } \\
\text { enriquecimento }\end{array}$} & $\mathbf{n}$ & $\begin{array}{c}\text { Peso seco de raíz } \\
\left(\mathbf{k g ~ h a}^{-1}\right)\end{array}$ & $\mathbf{C V ~ ( \% )}$ \\
\hline Sclerolobium paniculatum & 5 & $7.120 \pm 730$ & 23,0 \\
Acacia angustissima & 15 & $6.750 \pm 350$ & 19,8 \\
Inga edulis & 15 & $6.558 \pm 290$ & 16,8 \\
Acacia mangium & 15 & $6.322 \pm 350$ & 21,7 \\
Clitoria racemosa & 15 & $6.105 \pm 290$ & 18,5 \\
Controle & 5 & $6.061 \pm 780$ & 28,8 \\
\hline $\begin{array}{l}\text { Diferença mínima significativa } \\
(\mathrm{p}<0,1)\end{array}$ & 1.408 & \\
\hline
\end{tabular}

A análise do peso seco de raiz em função do espaçamento e independente das espécies leguminosas é apresentada na Tabela 5. Houve tendência de aumento do peso seco de raiz de mandioca com a diminuição do número de árvores plantadas por hectare. $\mathrm{O}$ menor valor de peso seco observado no espaçamento $1 \mathrm{~m} \mathrm{x} 1 \mathrm{~m}$ $\left(5.610 \pm 300 \mathrm{~kg} \mathrm{ha}^{-1}\right)$ foi estatisticamente menor que o peso seco obtido quando as árvores foram plantadas no espaçamento $2 \mathrm{~m} \times 2 \mathrm{~m}$. Os resultados observados para os espaçamentos $2 \mathrm{~m} \times 1 \mathrm{~m}$ e $2 \mathrm{~m} \times 2 \mathrm{~m}$, em valores absolutos, foram maiores que o controle, mas não foram estatisticamente diferentes. No espaçamento $1 \mathrm{~m}$ x $1 \mathrm{~m}$ havia na parcela experimental, o equivalente a $10 \mathrm{mil}$ árvores leguminosas e 10 mil plantas de mandioca por hectare. Essa grande concentração de plantas resultou em maior competição por recursos do solo e diminuição da produção de peso seco de raiz.

Tabela 5. Médias ( \pm erro padrão) e coeficientes de variação (CV) dos pesos secos de raízes de mandioca obtidos em consórcio com A. angustissima, C. racemosa, I. edulis, A. mangium e $S$. paniculatum plantadas para enriquecimento da vegetação de pousio em três espaçamentos.

\begin{tabular}{lccc}
\hline $\begin{array}{c}\text { Espaçamento de } \\
\text { plantio }\end{array}$ & n & $\begin{array}{c}\text { Peso seco de raiz } \\
\left.\mathbf{( k g ~ h a}^{-1}\right)\end{array}$ & CV (\%) \\
\hline 1 m x 1 m & 20 & $5.612 \pm 300$ & 24,1 \\
Controle & 5 & $6.061 \pm 780$ & 28,8 \\
2 m x 1 m & 25 & $6.660 \pm 220$ & 16,1 \\
2 m x 2 m & 20 & $7.143 \pm 200$ & 12,7 \\
\hline Diferença mínima & & 1.256 & \\
significativa (p $<0,1)$ & & &
\end{tabular}

A produção de raiz fresca de mandioca, de importante valor prático para o agricultor, pois permite estimar a quantidade farinha a ser produzida, também mostrou a mesma tendência do peso seco já mencionado anteriormente. As produções de raízes frescas de mandioca em função das espécies arbóreas plantadas e dos seus espaçamentos são mostradas nas Tabelas 6 e 7.

Tabela 6. Médias ( \pm erro padrão) e coeficiente de variação (CV) dos pesos frescos de raízes de mandioca obtidos em consórcio com diferentes leguminosas arbóreas plantadas para enriquecimento de vegetação de pousio nos espaçamentos $1 \mathrm{~m}$ x $1 \mathrm{~m}, 2 \mathrm{~m}$ x $1 \mathrm{~m}$ e $2 \mathrm{~m}$ x $2 \mathrm{~m}$.

\begin{tabular}{lrcc}
\hline \multicolumn{1}{c}{$\begin{array}{c}\text { Sistema de } \\
\text { enriquecimento }\end{array}$} & $\mathbf{n}$ & $\begin{array}{c}\text { Peso Fresco de raiz } \\
\left(\mathbf{k g ~ h a}^{-1}\right)\end{array}$ & CV (\%) \\
\hline $\begin{array}{l}\text { Sclerolobium } \\
\text { paniculatum }\end{array}$ & 5 & $23.920 \pm 1.690$ & 15,8 \\
Acacia angustissima & 15 & $22.800 \pm 945$ & 16,1 \\
Acacia mangium & 15 & $22.290 \pm 877$ & 15,2 \\
Inga edulis & 15 & $21.930 \pm 916$ & 16,2 \\
Controle & 5 & $21.200 \pm 1.510$ & 15,9 \\
Clitoria racemosa & 15 & $20.890 \pm 735$ & 13,6 \\
\hline $\begin{array}{l}\text { Diferença mínima } \\
\text { significativa }(\mathrm{p}<0,1)\end{array}$ & & 3.560 & \\
\hline
\end{tabular}

Tabela 7. Médias ( \pm erro padrão) e coeficientes de variação (CV) dos pesos frescos de raízes de mandioca obtidos em consórcio com A. angustissima, C. racemosa, I. edulis, A. mangium e $S$. paniculatum plantadas para enriquecimento da vegetação de pousio em três espaçamentos.

\begin{tabular}{lccc}
\hline $\begin{array}{l}\text { Espaçamento } \\
\text { de plantio }\end{array}$ & n & $\begin{array}{c}\text { Peso fresco de raiz } \\
\left(\mathbf{k g ~ h a}^{-1}\right)\end{array}$ & CV (\%) \\
\hline $1 \mathrm{~m}$ x 1 m & 20 & $20.060 \pm 803$ & 17,9 \\
Controle & 5 & $21.200 \pm 1.510$ & 15,9 \\
$2 \mathrm{~m}$ x 1 m & 25 & $22.487 \pm 582$ & 12,9 \\
$2 \mathrm{~m}$ x 2 m & 20 & $23.746 \pm 636$ & 12,0 \\
\hline Diferença mínima & 3.251 & \\
significativa $(\mathrm{p}<0,1)$ &
\end{tabular}

Os resultados de produção de raiz fresca de mandioca do presente trabalho são comparáveis com outros estudos realizados na mesma região. Num ensaio de enriquecimento de capoeira, usando Acacia auriculiformis, a exemplo do observado no presente trabalho, a maior população de árvores plantadas reduziu a produtividade da mandioca. Foram observados valores de $33 \mathrm{t} \mathrm{ha}^{-1}$ e $30 \mathrm{t} \mathrm{ha}^{-1}$ para as densidades de plantio de 2.500 e 10 mil árvores por hectare, respectivamente (Silva Júnior et al., 1998). 
As produtividades médias de raízes frescas do Município de Igarapé-açu, da região Bragantina (13 municípios incluindo Igarapé-açu) e do Estado do Pará são próximas aos índices nacionais, mas inferiores a do presente trabalho (IBGE, 2011) (Tabela 8).

Tabela 8. Produções de raiz fresca de mandioca $\left(\mathrm{kg} \mathrm{ha}^{-1}\right) \mathrm{em}$ função do tempo (anos) para o Brasil, Estado do Pará, Região Bragantina, PA, e Município de Igarapé-Açu, PA.

\begin{tabular}{lcccc}
\hline \multirow{2}{*}{ LocaIS } & \multicolumn{4}{c}{ Produção de mandioca $\left(\mathrm{kg} \mathrm{ha}^{-1}\right)$} \\
\cline { 2 - 5 } & $\mathbf{2 0 0 6}$ & $\mathbf{2 0 0 7}$ & $\mathbf{2 0 0 8}$ & $\mathbf{2 0 0 9}$ \\
\hline Brasil & 14.046 & 14.009 & 14.137 & 13.861 \\
Estado do Pará & 16.169 & 16.081 & 15.741 & 15.686 \\
Região Bragantina & 13.324 & 13.749 & 14.187 & 15.034 \\
Igarapé-Açu & 18.000 & 18.000 & 18.000 & 15.000 \\
\hline
\end{tabular}

O agricultor familiar amazônida que não tem a tradição de adubar a cultura da mandioca obtém, de um modo geral, produção de raízes variando de 15,82 $\mathrm{t} \mathrm{ha}^{-1}$ (Modesto et al., 2010) a $20 \mathrm{t} \mathrm{ha}^{-1}$ (Albuquerque \& Cardoso, 1983). As variações de produtividade na região podem ser atribuídas principalmente às diferentes variedades utilizadas e a qualidade do material selecionado para plantio, considerando maturidade do caule, aspecto fitossanitário e parte da planta mãe (Cardoso \& Kato, 1987).

\section{Conclusões}

A sobrevivência das árvores plantadas concomitantemente com milho e mandioca, em áreas de agricultura tradicional de corte-e-queima, não foi impactada negativamente pela presença das culturas agrícolas.

A produtividade da mandioca não foi afetada pela presença das árvores plantadas, independente do espaçamento de plantio.

É possível plantar árvores de crescimento rápido, a partir de roçado agrícola, com o objetivo de produzir biomassa e/ou madeira, entretanto, o espaçamento $1 \mathrm{~m}$ x $1 \mathrm{~m}$ deve ser evitado quando o objetivo principal for produzir alimento.

\section{Referências}

ALBUQUERQUE, M. de; CARDOSO, E. M. R. Utilização da mandioca na Amazônia. Belém, Embrapa - CPATU. 11p. (Embrapa - CPATU. Documentos, 25). 1983.
ALMEIDA, C. A.; VALERIANO, D. M., ESCADA, M. I. S., RENNO, C. D. Estimativa de área de vegetação secundária na Amazônia Legal Brasileira. Acta Amaz. 40(2): 289-301. 2010.

ALVES, R. N. B.; SOAVE, L. A.; GABY, Y. C. G.; VARANDA, D. de O.; ACOSTA, A. E.; CHAVES, J. R.; FRANÇA, M.F. BR 106 - milho para a agricultura familiar. Belém: Embrapa Amazônia Oriental. Folder. 2001.

BRIENZA JÚNIOR, S. Cordia goeldiana Huber (freijó) em sistema Taungya na região do Tapajós, Estado do Pará. Belém: EMBRAPA-CPATU. (EMBRAPA-CPATU. Circular Técnica, 33). 10 p. 1982.

BRIENZA JÚNIOR, S.; DUBOIS, J. C.; KITAMURA, P. C. Considerações biológicas sobre um sistema agrícola rotativo na região do Tapajós, Pará. Belém: EMBRAPA-CPATU. (EMBRAPA-CPATU. Boletim de Pesquisa, 50). 22 p. 1983.

BRIENZA JÚNIOR, S. Biomass dynamics of fallow vegetation enriched with leguminous trees in the Eastern Amazon of Brazil.Göttinger Beiträge zur Land- und Forstwinrtschaft in den Tropen und Subtropen. Heft 134. 133 p. 1999.

CARDOSO, E. M. R.; KATO, M. do S. A. Mandioca. Belém, Embrapa - UEPAE de Belém. 2p. (Embrapa - UEPAE de Belém. Recomendações Básicas, 4). 1987.

CASTRO, W. V. de; FARIAS NETO, J. T.; CAVALCANTE, E. da S. Efeito do espaçamento na produtividade de biomassa de taxi-branco (Sclerolobium paniculatum Vogel). Acta Amaz. 28(2): 141-146. 1998.

CATINOT, R. Sylviculture tropicale en forêt dense africaine. Perspectives d'aménagement (Fin). Bois et Forêt des Tropiques, Nogent-sur-Marne, (104):17-31. 1965.

DENICH, M. Untersuchungen zur Bedeutung junger Sekundärvegetation für die Nutzungssytemproduktivität im östlichen Amazonasgebiet, Brasilien. Göttinger Beiträge zur Land- und Forstwirtschaft in den Tropen und Subtropen 46. 265p. 1989.

FAO. Eucalyptus for planting. Rome: Food and Agriculture Organization of the United Nations. 1979. FAO Forestry and Forest Products Studies, n 11. 677 p. 1981.

GALVÃO, E. U. P.; CRAVO, M. da S.; NOGUEIRA, O. L.; SHIMIZU, M. K. Sistema bragantino para a agricultura familiar: passo a passo. Belém: Embrapa Amazônia Oriental, 2008. 38p.

HÖLSCHER D. Wasser- und Stoffhaushalt eines Agrarökosystems mit Waldbrache im östlischen Amazonasgebiet. Gött. Beiträge zur Land- und Forstwirtschaft in den Tropen und Subtropen 106. 133p. 1995.

IBGE. Instituto Brasileiro de Geografia e Estatística. 2011. Rio de Janeiro. Disponível em http://www.sidra.ibge.gov.br/bda/tabela/ protabl.asp? $\mathrm{c}=99 \& \mathrm{z}=\mathrm{t} \& \mathrm{o}=11 \& \mathrm{i}=\mathrm{P}($ acessado em $26 / 11 / 2011)$.

KATO, M. do S.A.; OLIVEIRA, C. de S.; OLIVEIRA, M. do S.S. de; KATO, O. R.; SANTANA, R.M. Agricultura sem queima: adaptando à realidade de agricultores familiares da comunidade São João Marapanim, PA. Belém: Embrapa Amazônia Oriental, 48 p. 2007. 
LOPES, O. M. N.; GALEÃO, R. R. Práticas de manejo do solo para produção agrícola familiar. Belém: Embrapa Amazônia Oriental, (Embrapa Amazônia Oriental. Documentos, 235). 23 p. 2006.

MARQUES, L. C. T.; YARED, J. A. G.; FERREIRA, C. A. P. Alternativa agroflorestal para pequenos produtores agrícolas em áreas de terra-firme do município de Santarém, Pará. Belém: EMBRAPA-CPATU. (EMBRAPA-CPATU. Boletim de Pesquisa, 147). 18 p. 1993.

MODESTO JÚNIOR, M.; ALVES, R. N. B.; SILVA, E. S. A. Produtividade de mandioca cultivada por agricultores familiares em áreas de mata de Paragominas, Pará. Amazônia: Ci. \& Desenv., Belém. 6(11): 179-190. 2010.

PEREIRA, O. G. A cultura do milho na Amazônia. Belém, Instituto de Pesquisas e Experimentação Agropecuárias do Norte. Série Fitotécnica. 1(5): 28 p. 1971.

SILVA, A. A. da; SOUSA FILHO, F. R. de; CORTELETTI, J.; PINTO, W. da S.; SILVEIRA, J. L. da; SILVA, S. R. M. da; KASPER, A.; MARQUES, U. M.; CAHETE, F. L. S. Historical dynamics of reproduction of agriculture in Igarapé-açu (Northeast of the State of Pará): a study focusing on agrarian systems. In: Lieberei, L.; Voss, K.; Bianchi, H. (eds). Proceedings of the Third SHIFT - Workshop. Bonn, BMB+F, Federal Ministry of Education and Research. Germany. 67-82p. 1998.
SILVA JÚNIOR, M. L.; VIELHAUER, K.; DENICH, M.; VLEK, P. L. G. Can tree enrichment of secondary vegetation and fire-free land preparation by cutting, chopping and mulching improve the following crops? In: Lieberei, L.; VOSS, K.; BIANCHI, H. (eds). Proceedings of the Third SHIFT - Workshop. Bonn, BMB+F, Federal Ministry of Education and Research. Germany. p. 113-117. 1998.

SOUZA, F. R. S. de; VELOSO, C. A. C.; POLTRONIERI, L. S.; ARAÚJO, S. M. B. de. Recomendações básicas para o cultivo de milho no Estado do Pará. Belém: Embrapa Amazônia Oriental, 20 p. (Embrapa Amazônia Oriental. Circular Técnica, 2). 1999.

STATISTICA for WINDOWS. Computer program manual, vers. 5.1. StatSoft, Inc, Tulsa, OK. 1998.

SYSTAT for WINDOWS. Computer program manual, vers. 5.0. SPSS Inc. 1994.

VIEIRA, I. C. G. Forest succession after shifting cultivation in Eastern Amazonia. University of Stirling, Scotland. 205p. (Ph.D. Thesis). 1996.

WATRIN, O. dos S. Estudo da dinâmica na paisagem da Amazônia Oriental através de técnicas de geoprocessamento. Instituto Nacional de Pesquisas Espaciais. São José dos Campos, SP. 153p. Dissertação de Mestrado. 1994.

YARED, J. A. G.; CARPANEZZI, A. A. Conversão de capoeira alta da Amazônia em povoamento de produção madeireira: o método do "recrû" e espécies promissoras. Belém, EMBRAPA-CPATU. (EMBRAPA-CPATU. Boletim de Pesquisa, 25). 27p. 1981. 
